\title{
Research on the Clinical Value of CT in Diagnosing Peripheral Lung Cancer Clinical Staging
}

\author{
Jun Sun ${ }^{a}$, Bo Mab ${ }^{b}$ He Liang', Li Lid, Fei Lin', Li Wang', * \\ The Third Affiliated Hospital of Qiqihar Medical University, Qiqihar City, Heilongjiang Province, 161000 \\ affeiji1981@sina.com, ’mabosunjun@163.com, ${ }^{c}$ lianghehei@163.com, d99701106@qq.com \\ e2891012105@qq.com, , *Corresponding author: shui.ying@163.com
}

Keywords: Multi-Slice Spiral CT; Peripheral Lung Cancer; clinical staging; application value

\begin{abstract}
Objective: To explore the value of multislice spiral CT in the diagnosis of peripheral lung cancer. Methods: A total of 128 patients with peripheral lung cancer who were admitted to our hospital from June 2015 to July 2017 were selected. All patients underwent multi-slice spiral CT examination. Their diagnostic results were compared with the results of histopathological examination. Results: Among the 88 lung cancer patients, adenocarcinoma accounted for 68.88\%, squamous cell carcinoma accounted for $28.38 \%$, and large cell carcinoma and small cell carcinoma accounted for $9.46 \%$. The incidence of lesions in each pathological type in each lobe was not statistically significant $(\mathrm{P}>0.05)$; There was no statistically significant difference in the size of lesions of each histopathological type $(\mathrm{P}>0.05)$. Conclusion: The diagnosis of peripheral lung cancer by multi-slice spiral CT can be used to make a preliminary qualitative judgment. It can be used as a common clinical screening method for peripheral lung cancer.
\end{abstract}

\section{Introduction}

Lmg Cancer, also known as Primary Bronchiogenic Carcinoma, refers to a malignant tumor that originates in bronchial mucosal epithelium. Its incidence is increasing year by year, and the incidence and mortality of lung cancer are in the midst of malignant tumors. In the first place, as the number of male smokers increases, the incidence of the disease in men gradually increases, develops rapidly and has a high degree of malignancy. About $80 \%$ of cases die within one year after diagnosis, and the five-year survival rate is less than $15 \%$ [1-2], a serious threat to human health. Clinically, the symptoms of early peripheral lung cancer are not obvious, and it has entered the late stage of obvious symptoms. In recent years, the application of multi-slice spiral CT has opened up a new way for the early diagnosis of peripheral lung cancer.

\section{Materials and Methods}

\subsection{General Information.}

From June 2015 to June 2016, 128 patients with peripheral lung cancer who were treated in our hospital met the relevant diagnostic criteria for peripheral lung cancer in Modern Oncology, including 50 males and 24 females; age 42 - 78 years old, average age (56.42 \pm 6.22$)$ years old.

\subsection{Methods.}

Patients were scanned using the Sonomam Definition AS 64-slice spiral CT (Siemens). The patient was instructed to take a supine position, and then a full lung scan was performed and the patient was held in a breathless state (while breathing still). Setting parameters: tube current $250 \mathrm{~m}$ A, tube voltage $120 \mathrm{k} \mathrm{V}$, scanning layer thickness $5.0 \mathrm{~mm}$. The range from the tip of the lung to the bottom of the ribs is the scanning range. If the patient is found to have pulmonary nodules during the examination, a thin layer reconstruction is performed. Parameters: thickness $1.25 \mathrm{~mm}$, interval $1.25 \mathrm{~mm}$. Lung window: Window position - 500HU, Window width 1500HU. Medial window: 40HU, 350HU. Scanned and intensified scans of suspicious lesions were performed with 
conventional doses. Dynamic contrast-enhanced scans were performed in 42 patients. The non-ionic contrast agent U-Visin (Bayer Healthcare Co., Ltd. Guangzhou Branch, ZhunGuozi H10970416) $80-100 \mathrm{ml}$ was used with a high-pressure syringe. Anterior bolus injection, $200 \mathrm{k} \mathrm{Pa}, 1.2 \mathrm{ml} / \mathrm{kg}$, rate 3.0-3.5ml/s. Enhanced scans were performed 15-20 seconds after injection. The acquired radiographic information was analyzed by two experienced radiologists and CT signs were recorded. This study uses the latest version of the TNM staging standard issued by the Unionfor International Cancer Control (UICC) in January 2009 as a general guideline. The CT examination report was jointly read by the four professional doctors of the imaging department and discussed together to obtain the same result as the result of the video. The controversial case was submitted to the Beijing expert for guidance. In preoperative staging, CTNM staging was performed by four senior doctors in thoracic surgery combined with all the auxiliary examination results. Pathological results PTNM were derived from the seventh edition of TNM.

\subsection{Observation Indicators and Technical Principles.}

Histopathological types were obtained by bronchoscopic biopsy or surgery, positioning and puncture, and the results of surgical pathological diagnosis of peripheral lung cancer and various spiral CT examinations were compared and analyzed. The stage of lung cancer established on the basis of TNM staging generally includes three stages, ie, a clinical stage based on all information obtained before the treatment is implemented or when no treatment has been determined; it is established on the pathological examination of resection specimens. Surgical pathology stages based on information; and staged after treatment in a multidisciplinary treatment regimen). Because the surgical pathological stage can describe the scope of the primary tumor and the regional lymph node metastasis in more detail, it has the highest value and is the gold standard for the staging of this study.

\subsection{Statistical Methods.}

SPSS 19.0 statistical software was used for analysis. Count data are expressed as percentages and cases. The x2 test was used for comparison between groups. Measured data were expressed as mean soil standard deviations for measurement data, and t-tests were used for comparison between groups. $\mathrm{P}<0.05$ was considered statistically significant.

\section{Results}

\subsection{Histopathological Classification.}

In 128 patients with lung cancer, adenocarcinoma accounted for 68.88\% (88/128), squamous cell carcinoma accounted for $21.88 \%$ (28/128), and large cell carcinoma and small cell carcinoma accounted for $9.46 \%(12 / 128)$.

\subsection{Histopathology and Lesion Location.}

The incidence of lesions in each pathological type in each lobe was not statistically significant $(\mathrm{P}>0.05)$.

Table 1 Relationship between lesion sites and histopathology

\begin{tabular}{lllcc}
\hline Lesion location & Adenocarcinoma & $\begin{array}{l}\text { Squamous } \\
\text { carcinoma }\end{array}$ & $\begin{array}{l}\text { cell } \\
\text { small } \\
\text { carcinoma }\end{array}$ & $\begin{array}{l}\text { and } \\
\text { cell total }\end{array}$ \\
\hline Top right & 26 & 11 & 0 & 37 \\
Right middle lung & 20 & 10 & 6 & 36 \\
Right lower lung & 15 & 4 & 1 & 20 \\
Left upper lung & 14 & 2 & 3 & 19 \\
Left lower lung & 13 & 1 & 2 & 16 \\
total & 88 & 28 & 12 & 128 \\
\hline
\end{tabular}


From Table 1, it can be seen that the lesions of different pathological types have no significant differences (X2=1.25, P>0.05). Explanation; There was no difference in the occurrence of lesions of different pathological types in each lobe.

The relationship between lesion size and histopathology, see Table 2.

Table 2 Relationship between lesion size and histopathology (cm)

\begin{tabular}{llcl}
\hline $\begin{array}{l}\text { Lesion } \\
\text { location }\end{array}$ & $\begin{array}{l}\text { Squamous } \\
\text { cell } \\
\text { carcinoma }\end{array}$ & Adenocarcinoma & $\begin{array}{l}\text { Large cell } \\
\text { and small cell } \\
\text { carcinoma }\end{array}$ \\
\hline Lesion size & $5.12 \pm 2.11$ & $3.45 \pm 1.51$ & $3.08 \pm 2.46$ \\
\hline
\end{tabular}

Table 2 shows that there is no significant difference in the size of lesions of different histopathological types $(\mathrm{F}=0.92, \mathrm{P}>0.05)$.

Sensitivity, specificity, and accuracy of CT staging, see Table 3.

Table 3 Analysis of sensitivity, specificity, accuracy, positive predictive value, and negative predictive value of CT stage in 88 adenocarcinomas (\%)

\begin{tabular}{llllll}
\hline CT staging & Sensitivity & Specificity & Accuracy & $\begin{array}{l}\text { Positive } \\
\text { predictive } \\
\text { value }\end{array}$ & $\begin{array}{l}\text { Negative } \\
\text { predictive value }\end{array}$ \\
\hline I、 II & $82.1(23 / 28)$ & $80.8(48 / 60)$ & $81.3(71 / 88)$ & $66.7(23 / 35)$ & $90.7(48 / 53)$ \\
IIIa & $78.0(35 / 45)$ & $65.9(28 / 43)$ & $72.2(63 / 88)$ & $71.0(35 / 50)$ & $73.7(28 / 38)$ \\
IIIb & $13.8(2 / 15)$ & $98.0(72 / 73)$ & $84.1(74 / 88)$ & $57.1(2 / 3)$ & $85.2(72 / 84)$ \\
\hline
\end{tabular}

\section{Discussion}

Lung cancer, which is primary bronchial carcinoma, mainly refers to malignant tumors that grow in bronchial epithelial tissues, and the incidence of this disease has become a rising trend. Currently, it is a disease with a high mortality rate in cancer [2-4]. The disease has a mortality rate of about $82 \%$ in the year after the diagnosis is established, and the five-year survival rate is relatively low. It has become one of the major killers of human health [5-6]. In recent years, the development and application of multi-slice spiral CT provide a favorable basis for the early diagnosis of peripheral lung cancer. Among the pathological types of peripheral lung cancer, adenocarcinoma is the most common [4-5],

With the rapid development of imaging technology, CT examination is gradually applied in the diagnosis of pulmonary diseases. Among them, multi-slice spiral CT has the advantages of high-density resolution, high spatial resolution, and fast scanning, showing the image characteristics of lung diseases. Clear, it is helpful to improve the diagnosis rate of thyroid diseases, and it is more common in lung disease examination [5-6].

The results of this study showed that the incidence of lesions in each pathological type was not significantly different in each lobe. There was no significant difference in the size of lesions in each histological type. It can be seen that the incidence and size of lesions in each pathological type are similar in the lung lobe. The histopathological classification of 128 patients with lung cancer was adenocarcinoma, squamous cell carcinoma, large cell carcinoma and small cell carcinoma. The typical spiral CT signs are short burrs, lobulated signs, pleural recession signs, and bundle vascular signs [7]. The results of this study show that there are significant differences in the CT signs found in each histopathological type. Among them, squamous cell carcinoma is the most common lobular sign, mostly accompanied by pneumonia and pleural effusion, mainly due to the slow development of squamous cell carcinoma. Large lesion growth, coupled with different tumor growth rates at various sites, it is prone to lobulation sign; adenocarcinoma is the most common burr sign associated with pleural effusion, atelectasis, analysis of the reasons for adenocarcinoma lesions 
easily lead The pleural membrane and the surrounding lung tissue are often associated with fibrosis.

In summary, the diagnosis of peripheral lung cancer patients with multi-slice spiral CT can be used to make a preliminary qualitative judgment, which can be used as a common clinical screening method for peripheral lung cancer.

\section{Acknowledgements}

Fund Project: 2016 Qiqihar City Science and Technology Bureau Prescriptive Scientific Research Project (Project Number: SFGG-201651)

\section{References}

[1] Wang Yunsheng, Zheng Haijun, Liu Hongyu et al. Clinical Value Analysis of Multislice Spiral CT in Diagnosis of Peripheral Lung Cancer [J]. Medical Imaging, 2015, 25(12): 2269-2271.

[2] Ma Daqing. CT diagnosis of peripheral lung cancer [J]. Chinese General Hospital, 2011, 10 (1): 69-70.

[3] Feng Yulan. A comparative study on the clinical value of X-ray and CT diagnosis of peripheral lung cancer [J]. Journal of Massage and Rehabilitation Medicine, 2011, 9(23):63-64.

[4] Sun Bowen, Liu Yongling, Yuan Hua. Diagnosis of Early Peripheral Lung Cancer with X-ray and CT [J]. Chinese Medical Engineering, 2012, 10(10):68-69.

[5] Ma Litang. CT diagnosis of peripheral lung cancer [J]. Medical Information, 2011, 2(24): 615-616.

[13] Yang Fan, Ma Yuehu, Chen Yuefang, et al. CT Diagnosis of Early Peripheral Lung Cancer in 96 Cases [J]. Journal of Practical Medical Techniques, 2012, 2(19): 152-153.

[6] Zhou Juan, Wang Lei, Zhao Fei. Application of multi-slice spiral CT in the diagnosis of peripheral lung cancer [J]. Modern Medical Imaging, 2017, 26(2): 367-368.

[7] Li Beiping. Clinical analysis of CT features of peripheral lung cancer [J]. Chinese and Foreign Medical Care, 2016, 35(13): 197-198. 